\title{
Hook Effect in Molar Pregnancy: A Report of Two Cases
}

\author{
${ }^{1}$ Shipra Kunwar, ${ }^{2}$ Hemprabha Gupta, ${ }^{3}$ Sipra Bagchi, ${ }^{4}$ Anushree Mittal
}

\begin{abstract}
Molar pregnancy is associated with very high levels of hCG in serum as well as in urine and therefore is an important marker in its diagnosis and follow-up. However, at times very high levels of hCG may be associated with falsely low or negative hCG levels because of Hook effect. Here, we report two cases molar associated with Hook effect.
\end{abstract}

Keywords: Molar pregnancy, HCG, Hook effect.

How to cite this article: Kunwar S, Gupta H, Bagchi S, Mittal A. Hook Effect in Molar Pregnancy: A Report of Two Cases. J South Asian Feder Obst Gynae 2014;6(1):49-50.

\section{Source of support: Nil}

Conflict of interest: None

\section{INTRODUCTION}

Gestational trophoblastic tumors are a range of tumors that arise from the trophoblast in early pregnancy, after an embryo has failed to develop. The degenerated trophoblast has a vesicular appearance and has been described to resemble a 'bunch of grapes.' These tumors produce large amounts of beta-human chorionic gonadotropin ( $\beta$-hCG). And, therefore, beta-hCG is the mainstay for diagnosis of gestational trophoblastic diseases. However, at times excessive beta-hCG production may produce a false negative result due to prozone phenomenon-also known as the 'hook effect'.

\section{CASE REPORTS}

\section{Case 1}

A 40 years old multiparous women presented to our hospital with chief complaints of passage of dark brown-colored discharge per vaginum for 1 month with no history of preceding amenorrhea. She also had history of abdominal distention with mild degree of lower abdominal pain and backache. She had no significant past medical history. She denied recent sexual activity and was not using any form of contraception.

\footnotetext{
${ }^{1}$ Associate Professor, ${ }^{2}$ Professor, ${ }^{3}$ Assistant Professor

${ }^{4}$ Junior Resident

1-4 Department of Obstetrics and Gynecology, Era's Lucknow Medical College and Hospital, Lucknow, Uttar Pradesh, India

Corresponding Author: Shipra Kunwar, Associate Professor Department of Obstetrics and Gynecology, Era's Lucknow Medical College and Hospital, Lucknow, Uttar Pradesh, India Phone: 7607133683, e-mail: shipra.kunwar@gmail.com
}

On examination, general condition was stable with pulse 88/min and BP 130/90 $\mathrm{mm} \mathrm{Hg}$. The cardiac examination was normal and the lungs were clear. She had a large, nontender abdominal mass corresponding to 22 weeks of gestation. Pelvic examination revealed a closed cervical os with blood mixed discharge and a 20 weeks sized uterus. A complete blood count, blood chemistries, pelvic ultrasound and quantitative serum $\beta$-hCG were performed. Urine pregnancy test was negative with $\beta$-hCG value of $5.4 \mathrm{mIU} / \mathrm{ml}$. Ultrasonography revealed enlarged uterus $(1036 \mathrm{ml})$ with heterogenous echogenic multiple small cystic content in the endometrial cavity with a large multiseptate $(10 \times 6 \mathrm{~cm})$ cyst in left ovary. Thyroid profile of the patient proved her to be euthyroid. In view of her $\beta$-hCG levels, a provisional diagnosis of degenerated submucosal fibroid was made and the patient was taken up for hysterectomy. However on cut section of uterus, vesicles were seen occupying whole of uterine cavity (Fig. 1). HPE revealed complete molar pregnancy. Prophylactic chemotherapy with methotrexate was given as the patient was not willing to come for follow-up.

\section{Case 2}

Patient aged 30 years para 3 with 3 live issues came to emergency of ELMCH with complain of bleeding per vaginum for 2 months off and on. Patient gave no history of prior ammenorrhea. On examination, patient was severely anemic and there was 34 weeks size mass per abdomen. On per speculum, examination bleeding was present. On investigations, her hemoglobin was $3.2 \mathrm{gm} \%$, urine pregnancy test was negative and ultrasound showed a large mass occupying

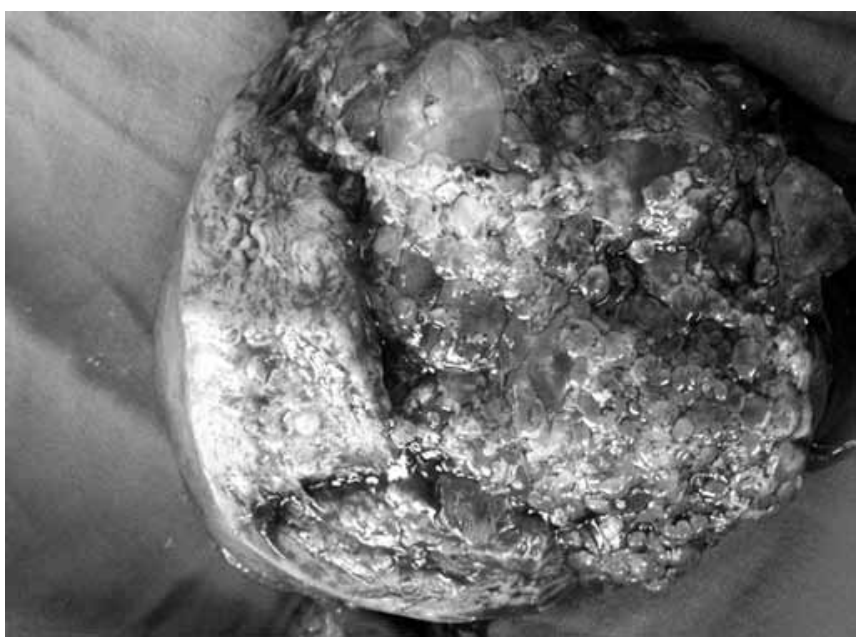

Fig. 1: Uterus showing vesicles after hysterectomy 
whole of uterus with cystic areas in between. Taking into account that there was no previous history of ammenorrhea and huge size of uterus and negative pregnancy test an initial diagnosis of degenerated fibroid was made. However, as there was a suspicion of molar pregnancy serum $\beta$-hCG done in dilutions revealed a value $1,60,000 \mathrm{mIU} / 1$. Patient was posted for suction evacuation after 3 units of blood transfusion. Histopathology revealed partial mole. Patient however did not report for follow-up.

\section{DISCUSSION}

Gestational trophoblastic disease is an uncommon diagnosis with incidence rates being highest in south-east Asian countries. Majority of women present with pelvic mass and vaginal bleeding along with a positive pregnancy test. Commonly used urine and serum pregnancy tests use immunometric sandwhich assays. ${ }^{1}$ These tests use antibodies directed against different parts of beta subunit of hCG.

Falsely low readings of hCG may occur with hydatidiform moles - a phenomenon called the 'high dose hook effect.' Hook effect was first described by Miles in $1975 .{ }^{2}$ The highdose hook effect occurs whenever there is an inordinate amount of substance being measured by an immunoassay, causing incomplete antibody-antigen complexes to form. Below a certain threshold concentration, the assay will reflect accurately the increasing concentration of the substance. Past the threshold concentration, when incomplete complexes begin to form, the assay will record falsely lower and lower readings as the concentration rises higher and higher. If one were to graph the readings of an immunoassay $v s$ increasing concentration of the measured substance, the graph would initially have a positive slope but would eventually peak or 'hook' and begin descending. Normally, results of an assay are read on the ascending part of the curve, but with large antigen excess, the results may hook and may therefore be read on the descending part of the curve. Dilution of a sample will return the 'reading' value to the true part of the curve, allowing accurate readings to resume. To eliminate the high-dose hook effect, the serum sample should be diluted before running the assay.

Another cause for abnormally low levels of serum $\beta$-hCG is production of abnormal forms of hCG like hCG-H (produced by invasive cytotrophoblast), nicked hCG, hyper/ hypoglycosylated $\mathrm{hCG}^{3}$ which might not be detected by regular hCG assays. Here, the use of DPC immunolite assay has been suggested. ${ }^{4}$

\section{CONCLUSION}

False-negative pregnancy tests have also been reported in ectopic pregnancy, multiple gestations and cancer. ${ }^{4}$ Negative or inconclusive results, in patients with high suspicion of pregnancy should be further evaluated by serum quantification of $\beta$-hCG and appropriate sample dilution. In an older patient with a molar pregnancy, increased vigilance for gestational trophoblastic neoplasia is required, and hysterectomy may be considered for those who have completed their families, or are noncompliant with follow-up. Although modern assay methods have much improved reliability, physicians should still be aware of the potential for false-negative urinary and serum $\beta$-hCG results due to the high-dose hook effect, especially in the setting of hydatidiform moles.

\section{REFERENCES}

1. Pang YP, Rajesh H, Tan LK. Molar pregnancy with false negative urine hCG: the hook effect. Singapore Med J 2010 Mar;51(3): 58-61.

2. Miles LE. Properties, variants and applications of the immunoradiometric assay method. Ric Clin Lab 1975;5:59-72.

3. Cole LA. Immunoassay of human chorionic gonadotropin, its free subunits, and metabolites. Clin Chem 1997;43:2233-2243.

4. Mehra R, Huria A, Gupta P, Mohan H. Choriocarcinoma with negative urinary and serum beta human chorionic gonadotropin (beta-hCG): a case report. Indian J Med Sci 2005 Dec;59(12):538-541. 